\title{
Quantitative evaluation of an epoxy resin dispersion by infrared spectroscopy
}

\author{
Shuichiro Yoshida \\ A new method for the quantitative analysis of an epoxy resin dispersion by infrared (IR) spectroscopy is described. The \\ absorbance ratio of various peaks in different areas of the IR spectra of a resin mixture, standardized to the IR absorbance of a \\ peak for aromatic ring hydrogens $\left(1593 \mathrm{~cm}^{-1}\right)$, is applied to evaluate the conditions for epoxy resin dispersion. The standard \\ deviation (s.d.) of the absorbance ratios in various regions of the spectra decrease as the resin mixing time increases, because \\ of changes in the dispersion conditions. This s.d. value is used as the threshold value for determining the proper dispersion \\ conditions. The threshold value enables quantitative evaluation of the epoxy resin; therefore, the proposed threshold value for \\ proper resin dispersion may be a very important factor for process control in epoxy resin manufacturing. \\ Polymer Journal (2014) 46, 430-434; doi:10.1038/pj.2014.15; published online 2 April 2014
}

Keywords: composite materials; dispersion; epoxy resin; infrared; rheology

\section{INTRODUCTION}

Epoxy resins are used in many industries as structural materials because they exhibit good mechanical and thermal behavior, high resistance to solvents and corrosive agents, adhesion to various substrates, and low shrinkage during curing. Composite materials comprising high-strength fibers and epoxy resins are used in the aerospace and automobile industries because they have low weight, high strength and high stiffness. ${ }^{1}$ In addition to these historical applications, fiber/epoxy resin composite materials are also being investigated for use in sound insulation. ${ }^{2}$

However, to achieve the high-performance properties of epoxy resins, uniform dispersion after mixing is important. Various types of basic epoxy resins, curing agents, accelerators and additives are combined to produce epoxy resins with specific performance characteristics, because, in some cases, simple compositions do not provide the necessary properties. ${ }^{3}$ If the dispersion of the resins and additives is not uniform, the material properties vary. For example, curing conditions may be insufficient if the curing agent or accelerator is not uniformly dispersed after mixing, which can lead to deterioration of the strength and heat resistance of the material. On the other hand, unexpected curing can occur if the mixing time is long. Therefore, a quantitative method for determining uniform epoxy resin dispersion, and thus proper mixing time, is necessary to produce consistently high-performing cured resins.

Visual evaluation and electron microscopy have been applied for the evaluation of epoxy resin dispersions in the past. ${ }^{3}$ However, visual evaluation is a qualitative method, and it is difficult to determine the dispersion conditions when the mixed resins and additives are of a similar color or colorless. Furthermore, sea-island structure evaluation using an electron microscope is a quantitative method; however, the evaluation area is very small and sample preparation and observation take a long time. Therefore, a quantitative and rapid method for resin dispersion evaluation is required.

Here a new method is described for the quantitative analysis of a mixed epoxy resin dispersion based on infrared (IR) spectroscopy. IR spectroscopy has previously been applied for the determination of the status of epoxy resin curing ${ }^{3-9}$ and degradation, ${ }^{10-13}$ but it has not yet been used to evaluate resin dispersion. This new method for the analysis of uniform dispersion by IR spectroscopy is quantitative because the absorbance ratios of the peak heights standardized to that of aromatic ring hydrogens are applied for the evaluation. Moreover, IR-attenuated total reflection spectroscopy enables rapid analysis. As a result, evaluating epoxy resin dispersions by IR-attenuated total reflection absorbance ratios is both quantitative and rapid.

\section{EXPERIMENTAL PROCEDURE}

\section{Materials}

Five types of base epoxy resins were used. The product names, general chemical names, and material suppliers are given in Figure 1. All materials were used as purchased. Bisphenol A epoxy resins BPAE1 and BPAE2 have the same basic chemical structure, but the number average molecular weight $\left(M_{\mathrm{n}}\right)$ of each is different (BPAE1, $M_{\mathrm{n}}=380$; BPAE2, $M_{\mathrm{n}}=900$ ). An aromatic amine used as the curing agent is described in Figure 2. No accelerators or additives were used.

The ingredients were mixed at $\sim 150^{\circ} \mathrm{C}$ at the weight percentages shown in Table 1. 


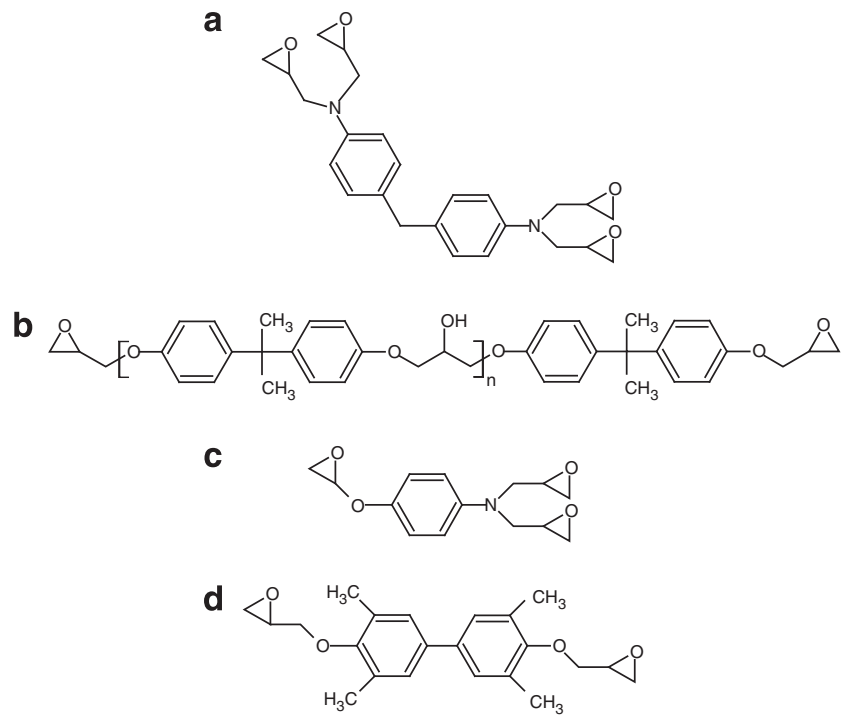

Figure 1 Chemical structures of the basic epoxy resins: (a) GDAE (glycidyl amine epoxy; Araldite MY721, Huntsman Corporation, The Woodlands, TX, USA), (b) BPAE1 (bisphenol A epoxy; jER 828, Mitsubishi Chemical Corporation, Tokyo, Japan; $M_{\mathrm{n}}=380$ ) and BPAE2 (bisphenol A epoxy; jER1001, Mitsubishi Chemical Corporation; $M_{n}=900$ ), (c) TPAP (triglycidyl-p-aminophenol; jER630, Mitsubishi Chemical Corporation), (d) TBPE (tetramethyl biphenolepoxy; YX4000, Mitsubishi Chemical Corporation).

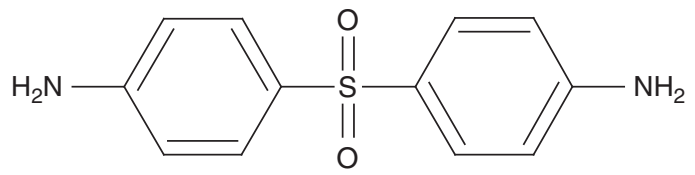

Figure 2 Chemical structure of the curing agent DDS(diaminodiphenyl sulfone; Aradur976-1, Huntsman Corporation.

Table 1 Resin mixtures evaluated for resin dispersion by IR spectroscopy

\begin{tabular}{|c|c|c|c|c|c|c|}
\hline \multirow[b]{2}{*}{ Sample no. } & \multicolumn{5}{|c|}{ Epoxy (wt\%) } & \multirow{2}{*}{$\begin{array}{c}\text { Curing agent }(\%, \\
\text { DDS }\end{array}$} \\
\hline & GDAE & $B P A E 1$ & BPAE2 & TPAP & TBPE & \\
\hline EP-a & 52 & 30 & 18 & - & - & 41 \\
\hline EP-b & 50 & - & - & 50 & - & 60 \\
\hline EP-c & 50 & 13.5 & 13.5 & - & 23 & 42 \\
\hline EP-d & 30 & - & - & 20 & 50 & 46 \\
\hline EP-e & 30 & 20 & - & - & 50 & 40 \\
\hline EP-f & 30 & - & 20 & - & 50 & 36 \\
\hline EP-g & 30 & 30 & 30 & - & 10 & 34 \\
\hline
\end{tabular}

Abbreviations: DDS, diaminodiphenyl sulfone; GDAE, glycidyl amine epoxy; TBPE, tetramethyl biphenolepoxy; TPAP, triglycidyl-p-aminophenol.

\section{Mixed resin preparation and dispersion evaluation by IR spectroscopy}

The measured resins and curing agent were placed in the chamber of a mixing machine connected to an oil heater. The heater was set at $150^{\circ} \mathrm{C}$ during mixing. The mixer had planetary-type blades, and the rotation speed was 260 r.p.m. The resin mixture was sampled with Pasteur pipettes at mixing times of $0,1,3,5,10$ and $30 \mathrm{~min}$ from 10 different areas in the mixer. By sampling the 10 areas shown in Figure 3, variations in the resin mixture at

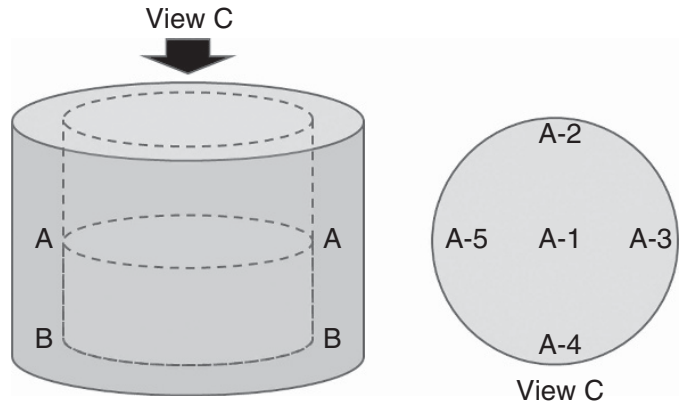

Figure 3 Sampling areas. Five areas were close to the surface of the mixed resin ( $A-1$ to $A-5)$, and five areas were near the bottom of the mixing chamber (B-1 to B-5). A full color version of this figure is available at Polymer Journal online.

Table 2 Infrared absorption peaks used for evaluation of the resin dispersion

\begin{tabular}{lrl}
\hline & Wave number \\
Peak no. & $\left(\mathrm{cm}^{-1}\right)$ & Peak identification \\
\hline P1 & 1610 & para-substituted benzene \\
P2 & 1504 & para-substituted benzene \\
P3 & 1288 & tertiary amine group \\
P4 & 1103 & sulfonated group \\
P5 & 904 & epoxy group \\
P6 & 826 & para-substituted benzene \\
P7 & 548 & para-substituted benzene \\
NA & 1593 & para-substituted benzene (standard for absorbance ratio)
\end{tabular}

Abbreviation: NA, not applicable.

various positions and depths were determined. Five of the sampling areas were close to the surface of the resin mixture (A-1 to A-5), and five were near the bottom of the mixing chamber (B-1 to B-5). Sampling was performed immediately after mixing stopped at each defined mixing time. Resin mixture EP-a in Table 1 was sampled at six mixing times $(0,1,3,5,10$ and $30 \mathrm{~min})$, and samples EP-b to EP-g were sampled at two mixing times (0 and $10 \mathrm{~min}$ ).

The IR absorbance spectra of the sampled resins were obtained by using an attenuated total reflection element (Spectrum One FT-IR Spectrometer, Perkin Elmer, Waltham, MA, USA). All measurements were collected from 4000$515 \mathrm{~cm}^{-1}$ at a $4 \mathrm{~cm}^{-1}$ resolution at room temperature in dry air. Sampled resins were stored inside the Pasteur pipettes until time for placement on the attenuated total reflection stage. The peak heights of the collected absorbance spectra for seven representative peaks (P1-P7 in Table 2) were standardized to the absorbance peak of aromatic ring hydrogens $\left(1593 \mathrm{~cm}^{-1}\right)$ to calculate the absorbance ratio $\left(1593 \mathrm{~cm}^{-1}\right.$ was selected as the standard absorbance ratio because aromatic ring hydrogens had a stable peak during the mixing procedure). These seven peaks were also identified in the IR spectra of glycidyl amine epoxy (GDAE), BPAE1, BPAE2, triglycidyl-p-aminophenol (TPAP), tetramethyl biphenolepoxy (TBPE), and diaminodiphenyl sulfone (DDS). The peak heights were determined with IR spectroscopy analysis software (Spectrum v.3.01) (Perkin Elmer).

The standard deviation (s.d.) of the absorbance ratios for the seven peaks of samples taken from the ten different areas of the mixing chamber at each mixing time was determined to investigate the change in the absorbance ratio with mixing time and to confirm that IR spectroscopy could be applied for the quantitative evaluation of the epoxy resin dispersion.

The formula for calculating the s.d., shown below, was used for the peaks at each identified wave number (P1-P7).

$$
\sigma_{m}=\sqrt{\frac{\Sigma\left(A_{n}-\bar{A}\right)^{2}}{n}}
$$


$\sigma_{m}=$ s.d. of each identified wave number of $m, A_{n}=$ each peak height at each identified wave number for spectra of different samples, $\bar{A}=$ average peak height at each identified wave number, $n=$ the number of peak data at each identified wave number, $\Sigma=$ sum of.

\section{RESULTS}

\section{IR absorption peak identification}

The IR spectra of GDAE, BPAE1, BPAE2, TPAP, TBPE and DDS are shown in Supplementary Figures S1-S6, respectively.

A representative IR absorption spectrum of mixed resin EP-a is shown in Figure 4. This spectrum was sampled from the A-1 area (Figure 3) after mixing for $10 \mathrm{~min}$. In this figure, the broad peaks from $3600-3200 \mathrm{~cm}^{-1}$ were attributed to hydroxyl groups. The sharp peaks from $3400-3300 \mathrm{~cm}^{-1}$, the peak at $1288 \mathrm{~cm}^{-1}$, and the peak at $1103 \mathrm{~cm}^{-1}$ were because of the tertiary amine, primary amine and sulfonated groups in DDS, respectively. The peaks attributed to aromatic ring hydrogens also appeared, near 1610, 1604, 1593, $1514,1508,1504,825,795$ and $548 \mathrm{~cm}^{-1}$. Sharp peaks for the aromatic ring hydrogens in GDAE appeared at 1610 and $1504 \mathrm{~cm}^{-1}$, whereas those in BPAE1 and BPAE2 appeared at 1504 and $826 \mathrm{~cm}^{-1}$. The peak at $1593 \mathrm{~cm}^{-1}$ because of the aromatic ring hydrogens in BPAE1, BPAE2 and DDS was used as the standard peak height for calculating the absorbance ratio. Finally, the epoxy groups in GDAE and in BPAE1 and BPAE2 appeared at $1231 \mathrm{~cm}^{-1}$ and $904 \mathrm{~cm}^{-1}$, respectively.

Variations in IR absorbance ratio with mixing time

Figure 5 shows the calculated s.d. of the absorbance ratios at $0,1,3,5$, 10 and 30 min mixing times for EP-a samples collected from the 10 different areas in Figure 3. The s.d. for all peaks at each mixing location decreased as the mixing time increased, and the s.d. drastically decreased after $5 \mathrm{~min}$ of mixing. At mixing times greater than $5 \mathrm{~min}$, the s.d. value was typically less than 0.03 .

\section{Validation of the maximum IR absorbance ratio of several epoxy} resin compositions before/after mixing

The calculated maximum s.d. of the absorbance ratios at mixing times of 0 and $5 \mathrm{~min}$, for samples collected from the 10 different areas in Figure 3, are shown in Figure 6. The s.d. drastically decreased after 5 min of mixing, even though the epoxy resin compositions were different. The maximum s.d. of the absorbance ratios were $\sim 0.6$ before mixing and decreased to $<0.04$ after 5 min of mixing. This result indicated that IR spectroscopy could be used as a successful method to evaluate the resin dispersion condition for various types of epoxy resins.

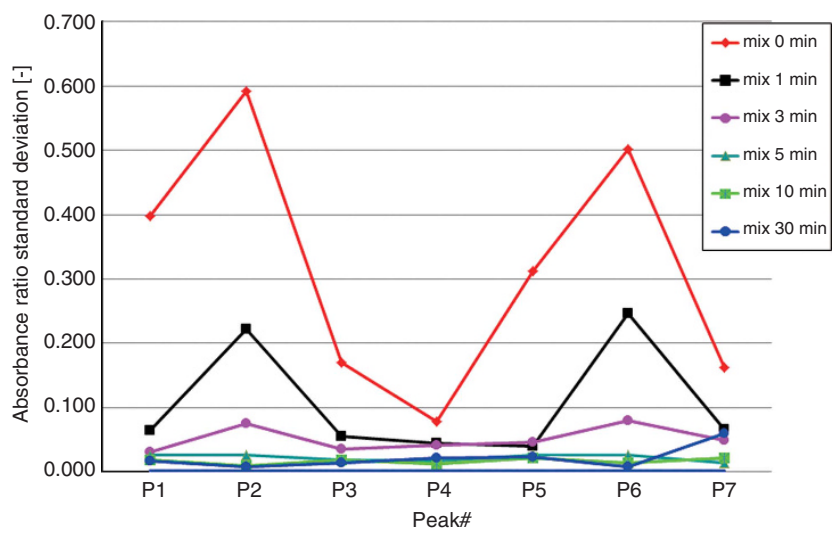

Figure 5 Standard deviation of the absorbance ratios of the seven representative peaks of mixed resin EP-a at each mixing time.

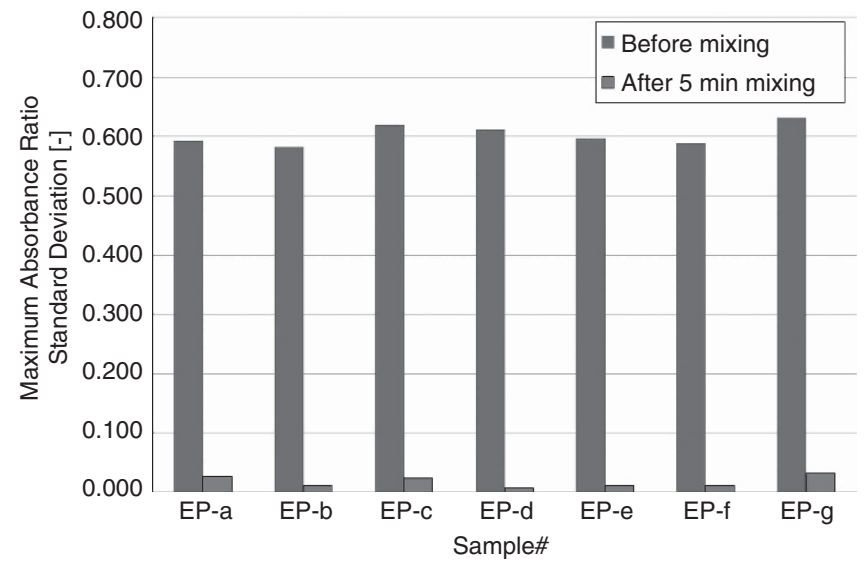

Figure 6 Standard deviation of the infrared absorbance ratio before/after mixing. A full color version of this figure is available at Polymer Journal online.

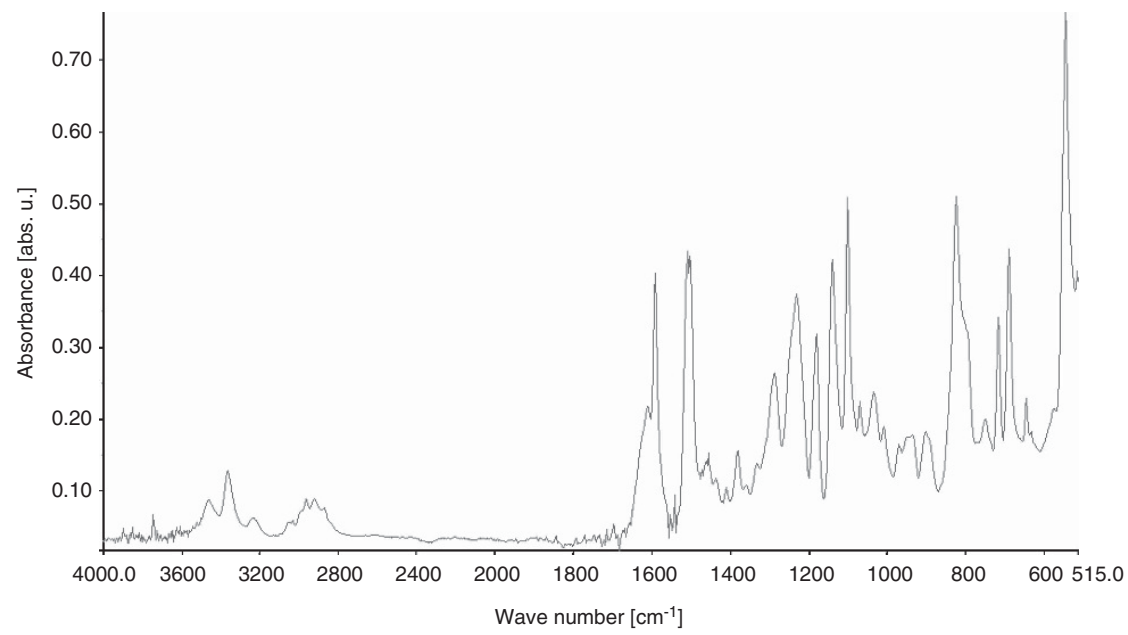

Figure 4 Representative infrared absorption spectrum of mixed resin EP-a at sampling position A-1 after mixing for 10 min. A full color version of this figure is available at Polymer Journal online. 


\section{DISCUSSION}

Determination of the threshold value for mixing time by IR spectroscopy

As can be seen in Figure 5, the s.d. of the absorbance ratios significantly varied for the different peaks when the mixing time was $5 \mathrm{~min}$ or less. Moreover, the s.d. of the absorbance ratios stabilized at low values for mixing times $>5 \mathrm{~min}$. Details of s.d. values at each mixing time are shown in Supplementary Table S1.

Figure 5 shows that the s.d. of the absorbance ratios were $<0.03$ after $5 \mathrm{~min}$ of mixing except for P7, which was 0.060 at $30 \mathrm{~min}$. Notably, the P7 s.d. increased from $0.021-0.060$ as the mixing proceeded from 10-30 min. Similarly, the s.d. of the absorbance ratios for peaks $\mathrm{P} 4$ and P5 increased with mixing from 10-30 min. Both P4 and P7 were attributed to DDS, while P5 was because of the epoxy groups. These results indicated that an unexpected epoxy curing reaction proceeded at long mixing times at $150{ }^{\circ} \mathrm{C}$, because the curing reaction caused the monomer and oligomer to structurally change to the polymer, which led to both a shift in these peak positions and a change in their peak heights because of broadening.

Although it cannot be definitively stated that a mixing time of 30 min leads to an unexpected curing reaction, the results suggest that such a long mixing time must be avoided. A time range from 5-10 min for this resin system is recommended on the basis of the stability of the s.d. of the absorbance ratios and the lack of any curing reaction. Moreover, on the basis of the current results, it is reasonable to assume that the mixing process is complete when the s.d. of the absorbance ratios at different sampling areas have values $<0.027$. The s.d. value of 0.027 was the maximum s.d. observed for mixing times between 5 and $10 \mathrm{~min}$. Thus, this value can be applied as the threshold value for determination of optimal mixing time.

\section{Variation in the s.d. of absorbance ratios}

Notably, there was variation in the s.d. for the different peaks, even at short mixing times. Specifically, from $0-5 \mathrm{~min}$ of mixing, P3 $\left(1288 \mathrm{~cm}^{-1}\right.$; tertiary amine group), P4 $\left(1103 \mathrm{~cm}^{-1}\right.$; sulfonated group) and P7 (548 $\mathrm{cm}^{-1}$; para-substituted benzene) had s.d. values for their absorbance ratios that were relatively lower than those of the other peaks (Figure 5). These three peaks occurred because DDS, a fine white powder at room temperature, was placed in the chamber after all of the epoxy resins were added to avoid any curing reaction while the materials were combined in the chamber. Hence, because of this order of addition, the DDS was in essence on the surface of the resin solution.

Samples were collected with Pasteur pipettes from 10 different sampling areas (Figure 3). DDS near the surface adhered to the sampled resin when samples were collected from areas B-1 to B-5. The DDS adhesion during sampling thus led to the stable presence of DDS in all sampled resins from all 10 areas, although the mixing process was incomplete. Therefore, the s.d. of the absorbance ratios for the peaks associated with DDS were lower than those of the other peaks.

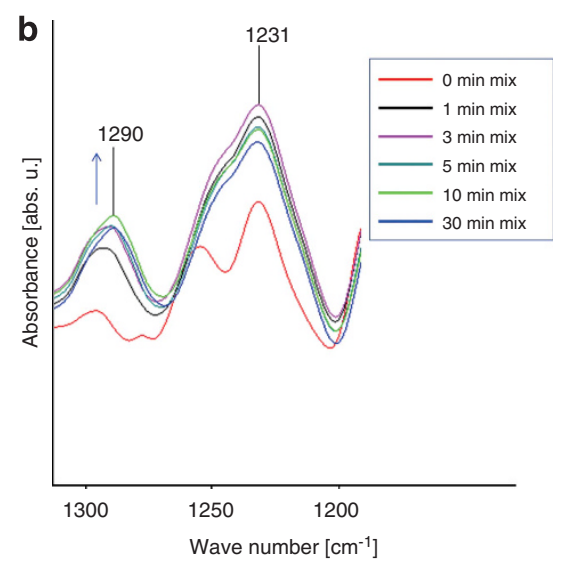

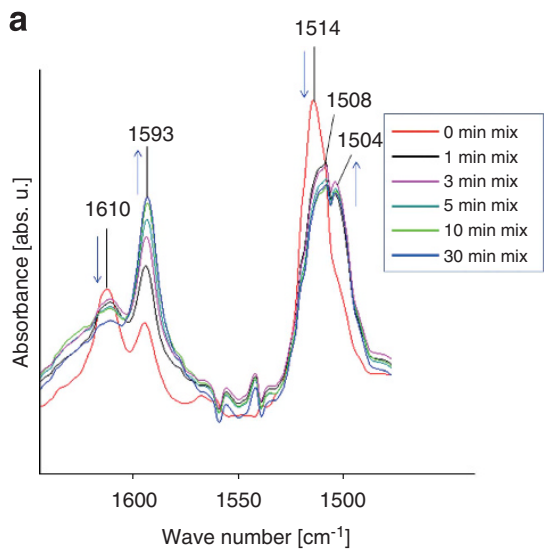

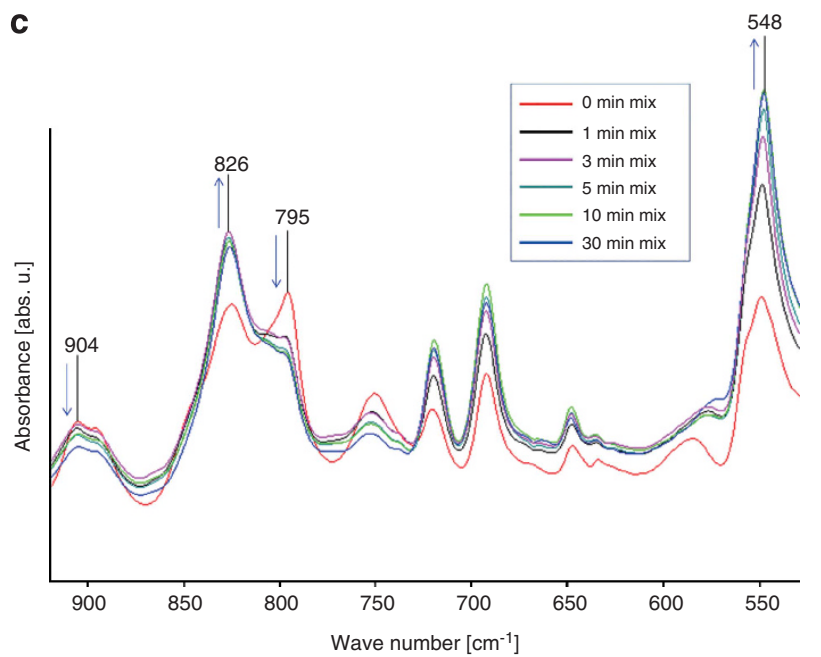

Figure 7 Enlarged infrared absorbance spectra for mixed resin EP-a at different mixing times: (a) $1640-1470 \mathrm{~cm}^{-1}$, (b) $1310-1190 \mathrm{~cm}^{-1}$ and (c) $890-540 \mathrm{~cm}^{-1}$ 


\section{Changes in IR absorbance spectra during mixing}

Figure 7 displays IR absorbance spectra for the EP-a resin mixture sampled from area A-1 (Figure 3) at different mixing times. Figures $7 \mathrm{a}-\mathrm{c}$ show the wave number regions $1640-1470 \mathrm{~cm}^{-1}$, $1310-1190 \mathrm{~cm}^{-1}$ and $890-540 \mathrm{~cm}^{-1}$, respectively. The peaks at 1593, 826 and $548 \mathrm{~cm}^{-1}$ because of the para-substituted benzene hydrogen of DDS increased as mixing progressed. This result indicates that the DDS powder was uniformly dispersed throughout the resin mixture. The same trend was observed for the DDS tertiary amine peak at $1290 \mathrm{~cm}^{-1}$.

On the other hand, the peaks at 1610, 1514 and $795 \mathrm{~cm}^{-1}$, which are because of the para-substituted benzene unit in GDAE, decreased as mixing proceeded because of the dispersion of GDAE, which results in the reduction of its concentration compared with that of BPAE1 and BPAE2. Moreover, the absorbance of the GDAE peak at $1514 \mathrm{~cm}^{-1}$ decreased and that of the peak for the para-substituted benzene in BPAE1 and BPAE2 at $1508 \mathrm{~cm}^{-1}$ increased.

Furthermore, the peaks at 1231 and $904 \mathrm{~cm}^{-1}$ because of the epoxy groups in GDAE and in BPAE1 and BPAE2 decreased as a result of mixing, particularly after $3 \mathrm{~min}$ of mixing, although the peak at $1231 \mathrm{~cm}^{-1}$ did initially increase $(0-1 \mathrm{~min}$ of mixing). This result indicates that ring opening of the epoxy group progressed during mixing because a mixing temperature of $150{ }^{\circ} \mathrm{C}$ was used. Therefore, mixing for an extended period is not recommended.

\section{Mixed condition judgment by IR spectroscopy for several types of epoxy compositions}

As can be seen in Figure 6, the s.d. of the absorbance ratios of the samples, collected from the 10 different areas in Figure 3, were all less than 0.04. Details of the s.d. values are shown in Supplementary Table S2. The maximum deviations of EP-b, EP-d, EP-e and EP-f in Table 1 after $5 \mathrm{~min}$ of mixing were $\sim 0.01$ when the TBPE content was $\sim 50 \mathrm{wt} \%$. TBPE was solid at room temperature but showed low viscosity, $0.2 \mathrm{P}$, at $150{ }^{\circ} \mathrm{C}$. The TBPE viscosity at high temperature led to rapid uniform mixing because lower-viscosity resin compositions are easier to mix than thick ones. In contrast, the deviations of EP-a, EP-c and EP-g after 5 min of mixing ranged from $\sim 0.02-0.03$, and these resin compositions contained $18-30 \mathrm{wt} \%$ BPAE2. BPAE2 had a viscosity of $8 \mathrm{P}$ at $150^{\circ} \mathrm{C}$, a value 40 times higher than that of TBPE; this viscosity discrepancy is the cause of the difference in s.d.

\section{CONCLUSION}

The s.d. of the absorbance ratios of seven peaks identified for samples collected from different areas of the mixing chamber decreased as mixing progressed. By evaluating the changes in the s.d. during mixing, it was possible to clearly determine the range of mixing times at which the lowest s.d. in absorbance ratios occurred. On the basis of these results, a threshold value for the s.d. was adopted for determining the proper mixing conditions. The resin dispersion was considered to be uniform when the s.d. of the absorbance ratios for samples taken from different areas in the mixing chamber were lower than this threshold value.

The proposed quantitative resin dispersion evaluation method by IR absorption spectroscopy was also successfully applied to several types of epoxy resin systems. The s.d. of the IR absorbance ratios drastically decreased as the mixing process approached uniform mixing.

1 Garton, A., McLean, P. D., Wiebe, W. \& Densley, R. J. Exposure of cross-linked epoxy resins to the space environment. J. Appl. Polym. Sci. 32, 3941-3957 (1986).

2 Liang, S., Xiu, Y. \& Wang, H. A research on sound insulation chracteristics and processing of the embedded and co-cured composite damping structures. J. Compos. Mater. 47, 1169-1177 (2013).

3 May, C. A. Epoxy Resins, Chemistry and Technology, Second Edition 1-550 (Marcel Dekker Inc., New York, 1988).

4 Musto, P., Abbate, M., Ragosta, G. \& Scarinzi, G. A study by Raman, near-infrared and dynamic-mechanical spectroscopies on the curing behavior, molecular structure and viscoelastic properties of epoxy/anhydride networks. Polymer 3703-3716 (2007).

5 Lee, H. \& Neville, K. Handbook of Epoxy Resins 1-183 (McGraw-Hill, New York, 1967).

6 Ellis, B. Chemistry and Technology of Epoxy Resins 1-71 (Blackie Academic and Professional, Glasgow, 1993)

7 Corcuera, M. A., Mondragon, I., Riccardi, C. C. \& Williams, R. J. Polymer networks derived from anhydride curing of tetraepoxides. J. Appl. Polym. Sci. 64, 157-166 (1997)

8 Nigel, A., St, J., Graeme, A. \& George Cure kinetics and mechanisms of a tetraglycidyl4,4'-diaminodiphenylmethane/diaminodiphenylsulphone epoxy resin using near i.r. spectroscopy. Polymer 33, 2679-2688 (1992).

9 Decker, C. \& Moussa, K. Kinetic study of the cationic photopolymerization of epoxy monomers. J. Polym. Sci. Part A Polym. Chem. 28, 3429-3443 (1990).

10 Mailhot, B., Morlat-Thérias, S., Bussiére, P. O. \& Gardette, J. L. Study of the degradation of an epoxy/amine resin, 2. Macromol. Chem. Phys. 206, 585-591 (2005).

11 Mailhot, B., Morlat-Thérias, S., Ouahioune, M. \& Gardette, J. L. Study of the degradation of an epoxy/amine resin, 1. Macromol. Chem. Phys. 206, 575-584 (2005)

12 Zhang, G., Pitt, W. G., Goates, S. R. \& Owen, N. L. Studies on oxidative photodegradation of epoxy resins by IR-ATR spectroscopy. J. Appl. Polym. Sci. 54, 419-427 (1994).

13 Chen, C. S., Bulkin, B. J. \& Pearce, E. M. New epoxy resins. III. Application of fourier transform IR to degradation and interaction studies of epoxy resins and their copolymers. J. Appl. Polym. Sci. 28, 1077-1091 (1983).

(c) (1)(2) This work is licensed under a Creative Commons Attribution-NonCommercial-ShareAlike 3.0 Unported License. The images or other third party material in this article are included in the article's Creative Commons license, unless indicated otherwise in the credit line; if the material is not included under the Creative Commons license, users will need to obtain permission from the license holder to reproduce the material. To view a copy of this license, visit http://creativecommons.org/licenses/by-nc-sa/3.0/ 